\title{
Morfología de esporas y sinangios en especies neotropicales del helecho Marattia (Marattiaceae)
}

\author{
María del Carmen Lavalle, Adriana Mengascini \& Mónica Rodríguez \\ Facultad de Ciencias Naturales y Museo, Universidad Nacional de La Plata. Argentina; mdelclavalle@hotmail.com, \\ adriamen@fcnym.unlp.edu.ar, mora_jade@hotmail.com
}

Recibido 10-I-2011. Corregido 12-IV-2011. Aceptado 16-V-2011.

\begin{abstract}
Spores morphology and synangia in neotropical fern species of Marattia (Marattiaceae). The Marattiaceae are represented by a small family of four to six genera that bear esporogenous structures of two types: sorus with free eusporangia in Angiopteris and Archangiopteris, and indurated synangium in Christensenia, Danaea and Marattia. Marattia is a pantropical genus of about eight to ten species in the paleotropic and seven to eight species in the neotropic. In order to describe the spores and sinangia morphology, this study analyzed the shape of the receptacles, and the position of the synangia, and evaluated the spores with SEM, of seven neotropical species of the genus Marattia: M. alata, M. cicutifolia, M. excavata, M. interposita, $M$. laevis, $M$. laxa y $M$. weinmanniifolia from several collections. The receptacles were fully developed in $M$. cicutifolia and $M$. laevis, and scarcely overelevated in the rest of the species. The synangium was ellipsoidal and had intramarginal to supramedial position in the laminae. The spores of Marattia were elliptic. Among the taxa, only monolete spores were found, with no trilete, aborted or deformed spores. The laesura was linear and reached about two of the total length of the spore. The perispore appears as a continuous thin layer deposited on the exospore according to its ornamentation in M. cicutifolia and M. laevis. It is smooth in M. alata, rugate in M. excavata and pustulate-rugate in two species: $M$. interposita and $M$. laxa. The exospore is echinate in $M$. cicutifolia and $M$. laevis and pustulate in the other species. In $M$. weinmannifolia spores produced by the same sinangium may have different ornamentation types. We concluded that, while the presence of ellipsoidal and superficial synangia and monolete spores aperture were generic traits, the micro and macro-ornamentation types of the perispores and exospores vary at specific level. Besides, macro-ornamentation can be bulliform (pustulate), a combination of bulliform and muriform types (pustulate-rugate), muriform (rugate-retate) and stelliform (echinate); finally, granular micro-ornamentation can be seen frecuently in perispores. Rev. Biol. Trop. 59 (4): 1833-1844. Epub 2011 December 01.
\end{abstract}

Key words: Marattia, neotropic, ornamentation, receptacles, sinangia, spores.

Marattiaceae, familia eusporangiada, incluye un número variable de géneros que difiere según los distintos autores y en el transcurso del tiempo. Copeland (1947) consideró seis géneros, Hill \& Camus (1986) cuatro, mientras que Rolleri et al. (2003) reconocieron cinco: tres paleotropicales, Angiopteris, Archangiopteris y Christensenia; uno neotropical, Danaea; y uno pantropical, Marattia. Más recientemente, Smith et al. (2006) consideraron cuatro, ya que incluyeron a Archangiopteris dentro de
Angiopteris; aunque un año más tarde, Christenhusz (2007) reconoció seis, porque separó a Macroglossum Copel. de Angiopteris.

El género Marattia cuenta con unas ocho a diez especies en el paleotrópico (Rolleri et al. 2001) y siete especies en el neotrópico según Lavalle (2003) u ocho según Christenhusz (2007) ya que separa $M$. chiricana Maxon de M. interposita, especies sinonimizadas previamente por Lavalle (2003). En cambio, Pérez-García (1995) considera la 
existencia de aproximadamente 60 especies en ambos trópicos.

La familia Marattiaceae presenta estructuras esporógenas de dos tipos: soros en Angiopteris y Archangiopteris, y sinangios en Christensenia, Danaea y Marattia. Según Bierhorst (1971) el único rasgo que diferencia sinangios de soros es el crecimiento de las paredes interesporangiales y sugiere que los soros son posteriores a los sinangios y que la condición de soros es secundaria y derivada de una condición sinangial. Para Millay (1978) soros y sinangios representan dos tendencias evolutivas paralelas en el orden; mientras que Liu et al. (2001) consideran que ambas clases de estructuras son igualmente antiguas y que pueden rastrearse hasta el Pérmico Superior.

Los sinangios de Danaea y Marattia se originan de manera similar a partir del meristema del receptáculo, que se encuentra hundido en el caso de Danaea y sobreelevado en Marattia (Bierhorst 1971). No hay estudios previos sobre receptáculos para ningún género de Marattiaceae, y sólo Hill \& Camus (1986) comparan someramente los receptáculos sobreelevados, llamados comúnmente "pedicelos", de M. laevis con los del fósil Scolecopteris Zenker (Millay 1979). A partir de ejemplares de Marattia con sinangios pedicelados, Smith (1842) segregó el género Eupodium Smith, aunque finalmente fueron agrupados en M. laevis.

El sinangio de Marattia fue llamado por Bierhorst (1971) esporocarpo e interpretado como dos sinangios enfrentados unidos en una sola estructura esporógena. El modo de origen de los esporangios individuales, tanto en soros como en sinangios es similar en todos los géneros, es decir, los esporangios se forman radialmente o en dos hileras enfrentadas, separadas por una delgada franja de tejido del receptáculo. Los sinangios bilaterales de contorno elíptico como los de Marattia habrían derivado de sinangios radiales de contorno circular como los de Christensenia (Taylor 1981).

Los estudios palinológicos en el orden Marattiales son escasos. Tryon \& Lugardon (1991) estudiaron las esporas de todos los géneros del orden mediante microscopía electrónica de barrido (MEB) y de trasmisión (MET), e incluyeron en ese trabajo cinco especies neotropicales de Marattia: M. cicutifolia, $M$. excavata, $M$. interposita, $M$. laevis y $M$. weinmanniifolia. Rolleri et al. (1998) compararon las esporas de varias especies de Angiopteris con las de otros géneros de Marattiaceae, incluyendo cuatro especies neotropicales de Marattia (M. alata, M. excavata, M. interposita y $M$. laevis). Encontraron esporas triletes pustuladas en Angiopteris, triletes equinadas en Archangiopteris y monoletes equinadas, en Danaea y Christensenia. En estos dos últimos géneros, la densidad, longitud y grado de fusión de las espinas son caracteres diagnósticos en el nivel específico. Posteriormente, Rolleri \& Lavalle (2001) estudiaron con MEB las esporas de ocho especies paleotropicales de Marattia analizando el valor diagnóstico de la micro y macro-ornamentación de perisporios y exosporios.

En el presente trabajo se ha analizado el aspecto de los receptáculos, la forma y la posición de los sinangios, y la micro y macroornamentación de los exosporios y perisporios de las esporas en las siete especies neotropicales de Marattia, con el fin de evaluar su significado taxonómico y poner a prueba el valor diagnóstico de su variación morfológica en la delimitación de las especies, ya conocidas por otros caracteres foliares externos (Lavalle 2005, 2007) e internos (Lavalle \& Mengascini 2007, Lavalle et al. 2008). Las especies estudiadas fueron: $M$. alata, $M$. cicutifolia, $M$. excavata, M. interposita, M. laevis, M. laxa y M. weinmanniifolia .

\section{MATERIALES Y MÉTODOS}

Se utilizó material de los siguientes herbarios: Museo Nacional de San José de Costa Rica (CR), Field Museum of Natural History (F), Herbarium Universitatis Florentinae (FI), Harvard University Herbaria (GH), Institute of Jamaica (IJ), División Plantas Vasculares, Facultad de Ciencias Naturales y Museo, Universidad Nacional de La Plata (LP), Herbarium, 
University of Michigan (MICH), Missouri Botanical Garden (MO), New York Botanical Garden (NY), Jardin Botânico do Rio de Janeiro (RB), Instituto de Botánica "Darwinion", San Isidro (SI) y United States National Herbarium, Botany Department, Smithsonian Institution (US), citados de acuerdo con Holmgren et al. (1990).

Marattia alata: Cuba: Oriente: Slopes of La Bayamesa, crest of the Sierra Maestra near Aserradero San Antonio de los Cumbres, Morton 9302 (US). Jamaica: Morce's Gap: Hopkins s/n (1381800 F).

Marattia cicutifolia: Brasil: San Paulo: Serra da Bocaina, Luederwaldt 20976 (NY); Santa Catharina: Sertaô do Lagoa, Rohr 1036 (US); Ribeirâo do Ouro, Brusque, Reitz 3543 (IJ, RJ, US). Río de Janeiro: Corcovado, Brade 8547 (NY).

Marattia excavata: Costa Rica: Puntarenas: $5 \mathrm{~km} \mathrm{~S}$ of San Vito de Java, 1-4km SW of station, vicinity of biological field station at Finca Wilson, Mickel 3119 (LP, US). El Salvador: Santa Ana: Nebuloso de Montecristo, camino al Trifinio, Villacorta et al. 1054 (MO). Guatemala: El Quiché: Valley of Rio de las Violetas, north of Nebaj, Proctor 25261 (IJ, US); Quezaltenango: Slopes of Volcan de Zunil, at and above Aguas Amargas, Standley 65461 (F). Honduras: Ocotepeque: Cordillera Merendón, vicinity El Portillo, Molina $R$. 30931 (F). México: Chiapas: on the Southeast side of Volcán Tacaná above Talquian, Breedlove \& Smith 31624 (MICH, MO, NY). Nicaragua: Granada: Volcán Mombacho, east side, near summit above Finca Las Delicias, Bruce Nelson 7545 (GH).

Marattia interposita: Costa Rica: Limón: Cordillera de Talamanca, Cerro Bitarkara, Reserva Biol. Hitoy Cerece, Herrera 2489 (CR). Guatemala: Quetzaltenango: Fuentes Georginas, Western slope of Volcán de Zunil, Standley 67512 (F). Nicaragua: Granada: trade winds side of Volcan Mombacho, Atwood, Jr. \& Neill AN188 (US). Panamá: Chiriquí, Cuesta de Las Palmas, S slope of Cerro de la Horqueta, Maxon 5525 (US).
Marattia laevis: Argentina: Misiones: Gral. Belgrano, ruta Nac. 101, Salto Andresito, Guaglianone et al. 2975 (SI). Bolivia: Santa Cruz: Williams 1289 (NY). Brasil: San Pablo: Campos do Jordâo, 40km North-NorthWest of Sâo José dos Campos, Tryon \& Tryon 6919 (F, GH). Colombia: Quindio: Salento, La Línea, Estación Piscícola Navarco, Madriñán \& Betancur $1317(\mathrm{GH})$. Costa Rica: Heredia: Volcán Barba, $\mathrm{S}$ of the principal peak above Porrosatí, Lellinger 1709 (F, US). Ecuador: Carchi: Cerro Golondrinas, upper Rio Gualpí watershed, Boyle \& Hibbs 2354 (MO). Perú: Huanuco: Churubamba, Mount Santo Toribio, trail Puente Durand to Éxito, Mexia 8250 (F, GH, MICH, NY, US). República Dominicana: Santiago: Cordillera Septentrional, 13 air km NW of Santiago at the peak of Loma Diego de Ocampo, Mickel \& Zanoni 9223 (NY). Venezuela: Trujillo: Boconó, 22km. SE of Boconó, road to Guaramacal, Smith 1505 (MO).

Marattia laxa: Honduras: Comayagua: El Portillo, Montaña Comayagua, Hazlett 2493 (F). México: Alto Lucero: Cerro de la Cima, entre La Sombra y Rancho Nuevo, Castillo \& Vázquez 1528 (F); Puebla: Curva de los Millones, near Teziutlan, Riba 299 (GH).

Marattia weinmanniifolia: México: Chiapas: eastern ridge of Chiapas Highlands, $3 \mathrm{~km}$ $\mathrm{N}$ of Pueblo Nuevo, Roe et al. 1238 (MICH); Guerrero: estribaciones suroccidentales del Cerro Teotepec, $2 \mathrm{~km}$ al NE del Campamento El Gallo, Rzedowski \& Mc Vaugh 141 (LP, $\mathrm{MICH}, \mathrm{NY}$ ).

Cortes transversales de receptáculos y sinangios: Las pínnulas y pinnúlulas se hidrataron con agua y unas gotas de detergente en estufa a $30^{\circ} \mathrm{C}$; se aclararon con hidróxido de sodio al 5\% acuoso entre $10-40 \mathrm{~min}$; se blanquearon con hipoclorito de sodio 1:1 en agua. Luego de un proceso corto de deshidratación con alcoholes (D'Ambrogio de Argüeso 1986) se incluyeron en "Paraplast Plus". Se cortaron con micrótomo tipo Minot en espesores de 7-10um y se tiñeron con safranina-verde rápido al $2 \%$ en éter monometílico del etilenglicol según Gurr (1965). Se ilustraron con un 
microscopio Wild M-20 equipado con cámara de dibujo.

Esporas: Para el estudio con MEB se tomaron de las láminas sinangios cerrados, se colocaron sobre porta-muestras de metal $(\mathrm{Cu}-$ $\mathrm{Zn}$ ) con cinta adhesiva doble cara y se abrieron Se realizaron varias preparaciones de cada ejemplar esterilizando el instrumental cada vez. Los restos de paredes sinangiales se retiraron del porta-muestras con un pincel fino. Las esporas, sin tratamiento previo, fueron recubiertas con oro bajo vacío con un metalizador Jeol JFC-1100 (fine coat ion sputter). Las fotografías se tomaron en el Servicio de Microscopía Electrónica de Barrido de la Facultad de Ciencias Naturales y Museo de La Plata con un microscopio Jeol JSM-T100. Se utilizaron los siguientes aumentos: x $750(15 \mathrm{kvol})$ para fotos panorámicas, x 5000 (15kvol) para esporas individuales, x $10000(25 \mathrm{kvol})$ para detalles de la ornamentación.

Las medidas de esporas son el promedio de 25 esporas por muestra, tomadas con un microscopio Wild M-20 con ocular de medida.

La terminología utilizada para la descripción de las esporas es la propuesta por Lellinger (2002).

\section{RESULTADOS}

Estructuras esporógenas (Fig. 1 y 2): En las especies neotropicales los receptáculos tienen contorno elíptico en vista superficial, presentan una epidermis con células de contorno poligonal (Fig. $1 \mathrm{~A}$ ), excepto en M. alata que son papilosas a la madurez (Fig. 1 B). Los receptáculos están apenas sobreelevados dos a

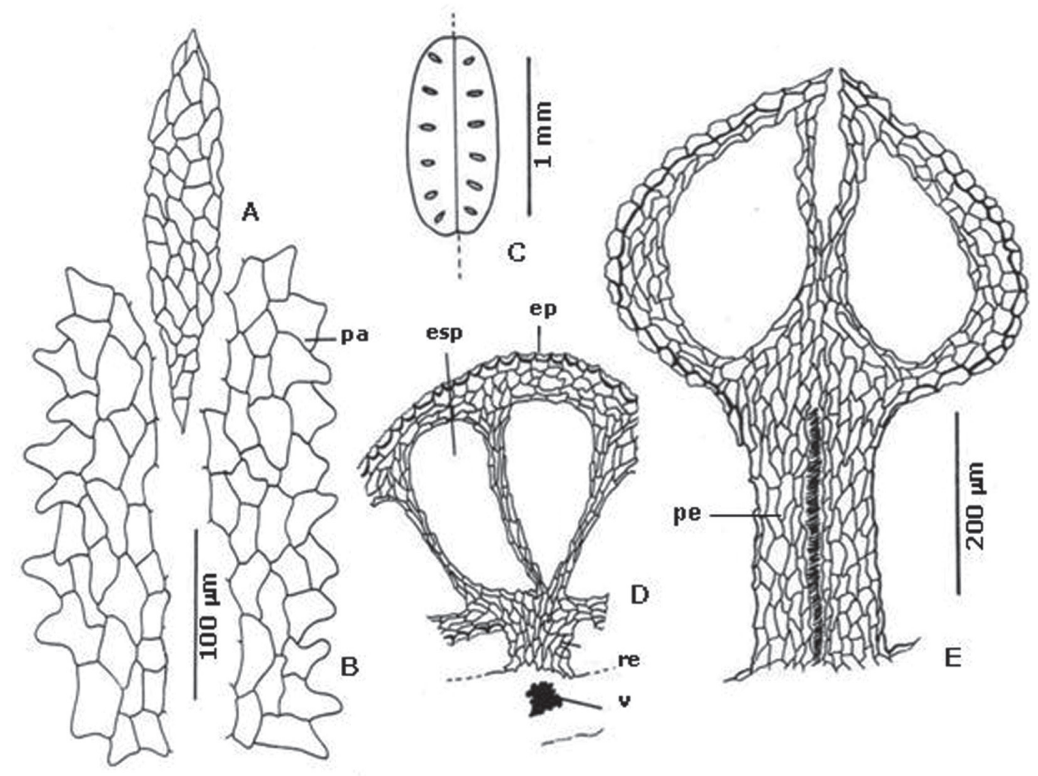

Fig. 1. Receptáculos y sinangios de Marattia. (A-D) M. alata. (A) Receptáculo juvenil en superficie. (B) Márgenes papilosos del receptáculo adulto, en superficie. (C) Sinangio. (D) Hemisinangio con dos esporangios. (E) M. laevis, corte longitudinal de un sinangio a lo largo del pedicelo. Las abreviaturas son: ep: epidermis, esp: esporangio, pa: papila, pe: pedicelo, re: receptáculo, v: vena.

Fig. 1. Receptacles and synangia in Marattia. (A-D) M. alata. (A) Juvenile receptacle in surface view. (B) Papillose margin of the adult receptacle, in surface view. (C) Synangium. (D) Hemisinangium with two sporangia. (E) M. laevis, longitudinal section of one synangium across the pedicel. The abbreviations are: ep: epidermis, esp: sporangium, pa: papilla, pe: pedicel, re: receptacle, v: vein. 

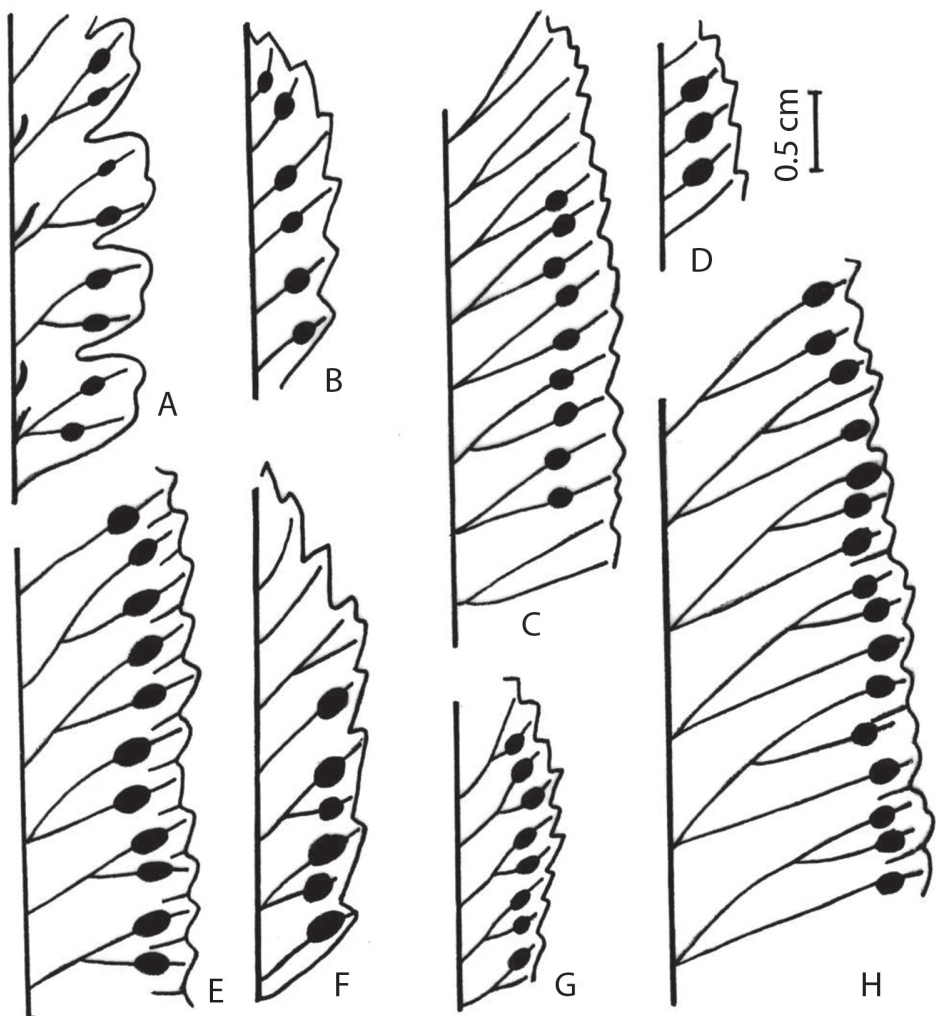

Fig. 2. Posición de los sinangios de Marattia. (A-D): supramedial en M. laevis (A-B); M. cicutifolia (C); M. weinmanniifolia (D). (E-H): Intramarginal en $M$. interposita (E); M. alata (F); M. excavata (G); M. laxa (H).

Fig. 2. Position of the synangia in Marattia. (A-D): Supramedial in M. laevis (A-B); M. cicutifolia (C); M. weinmanniifolia (D) (E-H): Intramarginal in M. interposita (E); M. alata (F); M. excavata (G); M. laxa (H).

cuatro capas celulares por encima de la epidermis de la lámina en $M$. alata, $M$. excavata, $M$. interposita, M. laxa y M. weinmanniifolia. En M. cicutifolia y M. laevis los receptáculos son elevados, hiperdesarrollados, crasos y vascularizados, y han sido llamados generalmente pedicelos. En M. laevis los receptáculos presentan su máximo desarrollo, alcanzan entre $0.7-1 \mathrm{~mm}$ de altura, con un haz vascular que llega hasta el sinangio (Fig. 1 E).

En Marattia, el sinangio (Fig. 1 C) se abre en dos mitades a través de una hendidura paralela a la vena sobre la cual se encuentra. Cada hemisinangio, llamado comúnmente "valva", incluye 12-20 eusporangios fusionados, denominados lóculos en las floras regionales, término más bien angiospérmico, que debería reemplazarse por el de esporangios. En un ejemplar de M. laevis del NE de Argentina los hemisinangios totalmente abiertos se disponen en un plano, tomando un aspecto cupuliforme.

La epidermis del sinangio presenta engrosamientos desiguales en " $U$ " con paredes externas muy delgadas (Fig. 1 D-E). La pared externa tiene cuatro a cinco capas celulares, como un eusporangio individual. Las paredes interesporangiales son delgadas y tienen dos a tres capas celulares de espesor (Fig. 1 D).

Los sinangios tienen aproximadamente 1.6-2.1mm de largo, son elipsoidales, de contorno elíptico ancho en especies neotropicales, y elíptico angosto en especies paleotropicales. 
La posición en las pínnulas o pinnúlulas es intramarginal a medial. Son intramarginales en M. alata (Fig. 2 F), M. excavata (Fig. 2G), M. interposita (Fig. 2 E) y M. laxa (Fig. 2 H) y supramediales en $M$. cicutifolia (Fig. 2 C), M. laevis (Fig. 2 A-B) y M. weinmanniifolia (Fig. 2 D).

Esporas (Fig. 3-6, Cuadro 1): En las especies neotropicales de Marattia las esporas son siempre monoletes y no se encuentran esporas triletes ni aletes. La lesura es poco marcada y en vista proximal tiene una longitud aproximadamente igual a la mitad del diámetro mayor de la espora. Las esporas tienen forma elíptica en vista polar y reniforme en vista ecuatorial. La forma y el tamaño resultaron constantes y nunca se observaron esporas abortadas, deformadas o gigantes (Fig. 3 A-D, Fig. 6 A).
Se encontraron tres clases de ornamentación: buliforme, muriforme y esteliforme y los tipos: pustulados, rugados, retiados y equinados. La macro-ornamentación puede ser igual o diferente en exosporio y perisporio, y es frecuente, como en las especies paleotropicales, la presencia de micro-ornamentación granular más o menos nítida en el perisporio, rara vez presente en el exosporio.

Las medidas y la ornamentación de las esporas para cada especie se detallan en el Cuadro 1.

Perisporio - Es delgado, sin micro-ornamentación y se deposita siguiendo los procesos espiniformes del exosporio, en M. cicutifolia y $M$. laevis. En M. cicutifolia se interrumpe en la base de las espinas (Fig. 4 C, D) mientras que en M. laevis (Fig. 4 A, B) se deposita en forma continua y sólo esporádicamente se interrumpe.


Fig. 3. Esporas de Marattia en vista panorámica. (A-B) Esporas equinadas, (A) M. laevis de Costa Rica, Lellinger 1709 (US). (B) M. cicutifolia de Brasil, Rohr 1036 (US); (C-D) Esporas pustuladas. (C) Esporas pustulado-retiadas en M. laxa de México, Castillo \& Vásquez 1528 (F). (D) Esporas pustulado-rugadas en M. interposita de Panamá, Maxon 5525 (US). Fig. A-D: $x 750$.

Fig. 3. Spores of Marattia in panoramic view. (A-B) Echinate spores, (A) M. laevis of Costa Rica, Lellinger 1709 (US); (B) M. cicutifolia of Brasil, Rohr 1036 (US); (C-D) pustulate spores, (C) Pustulate-retate spores in M. laxa of México, Castillo \& Vásquez 1528 (F); (D) Pustulate-rugate spores in M. interposita of Panamá, Maxon 5525 (US). Fig. A-D: x 750. 
CUADRO 1

Dimensiones y ornamentación de las esporas en especies neotropicales de Marattia

\begin{tabular}{|c|c|c|c|c|}
\hline \multirow{2}{*}{ Especie } & \multicolumn{2}{|c|}{ Dimensiones (en mm) } & \multicolumn{2}{|c|}{ Ornamentación } \\
\hline & Largo & Ancho & Exosporio & Perisporio \\
\hline M. alata & $18.6(22) 24.3$ & 11.8 (14) 16.5 & Pustulado & $\begin{array}{l}\text { Delgado con micro- } \\
\text { ornamentación granular }\end{array}$ \\
\hline M. cicutifolia & $24.1(27.7) 31$ & 15.5 (18.4) 20.7 & $\begin{array}{l}\text { Equinado con espinas } \\
\text { cortas }\end{array}$ & $\begin{array}{l}\text { Liso, se interrumpe en la base } \\
\text { de las espinas }\end{array}$ \\
\hline M. excavata & 20.7 (23.4) 27.6 & 13.8 (15) 17.2 & Pustulado & Rugado \\
\hline M. interposita & 20.7 (23.6) 25.9 & 10.3 (13.6) 15.5 & $\begin{array}{l}\text { Pustulado con micro- } \\
\text { ornamentación granular } \\
\text { gruesa }\end{array}$ & $\begin{array}{l}\text { Pustulado-rugado con micro- } \\
\text { ornamentación granular }\end{array}$ \\
\hline M. laevis & $24.1(25.2) 27.6$ & $13.8(17.4) 19$ & $\begin{array}{l}\text { Equinado con espinas } \\
\text { largas }\end{array}$ & $\begin{array}{l}\text { Liso, se deposita en forma } \\
\text { continua siguiendo las espinas } \\
\text { del exosporio }\end{array}$ \\
\hline M. laxa & 19 (21) 24.1 & 10.3 (13) 15.5 & Pustulado & Pustulado-rugado \\
\hline M. weinmanniifolia & $24.1(25.7) 28$ & 15.5 (16.7) 19 & Pustulado & $\begin{array}{l}\text { Retiado y rugado con micro- } \\
\text { ornamentación }\end{array}$ \\
\hline
\end{tabular}

En M. alata forma una capa continua que es rugada a casi lisa, con micro-ornamentación granular algo difusa (Fig. 5 E, F). En M. excavata el perisporio es rugado, los muros son cortos, libres y no hay micro-ornamentación (Fig. 6 A y C).

En M. weinmanniifolia hay dos tipos de perisporio: retiado, con muros anchos y redondeados que se anastomosan formando aréolas sin micro-ornamentación (Fig. $6 \mathrm{~J}, \mathrm{~K}$ ), y rugado, con micro-ornamentación granular gruesa y nítida en toda la superficie (Fig. 6 I). Ambos se encuentran en esporas adultas normales producidas por el mismo sinangio.

En M. interposita y M. laxa el perisporio es pustulado rugado con muros libres o parcialmente anastomosados sin formar aréolas y bulas aisladas; en M. laxa (Fig. 6 E, G, parcial en F) los muros son altos, anchos y curvos, sin micro-ornamentación; mientras que en $M$. interposita (Fig. 5 B, C) son muy bajos, y a veces con aspecto de pliegues, cubiertos con micro-ornamentación granular.

Exosporios - El exosporio es pustulado en M. alata (Fig. 5 D, parcial en E, F), M. excavata (Fig. 6 B), M. interposita (Fig. 5 A, parcial en C), M. laxa (Fig. 6 D) y M. weinmanniifolia (Fig. $6 \mathrm{H}$ ) y es equinado en M. cicutifolia (Fig. 4 D) y M. laevis (Fig. 4 B).

Los exosporios pustulados presentan bulas bajas, más o menos dispersas y muy uniformes. Todos los exosporios descritos carecen de micro-ornamentación, excepto en $M$. interposita que presenta una micro-ornamentación granular gruesa y muy dispersa (Fig. $5 \mathrm{~A}, \mathrm{C}$ ).

En M. laevis (Fig. 4 A, B) y M. cicutifolia (Fig. 4 C, D) el exosporio equinado presenta espinas espaciadas. Las espinas de M. laevis son cónicas largas (cinco a seis veces más largas que el ancho basal), con ápice agudo, romo o bífido, base redondeada y cuerpos erectos o distalmente curvos. Las espinas de M. cicutifolia son cónicas cortas (dos a tres veces más largas que el ancho basal), con ápices agudos y delgados, más raramente romos, y el cuerpo puede ser curvo o erecto y anillado.

\section{DISCUSIÓN}

La aparición de sinangios cupuliformes de margen entero y con la mayoría de los esporangios dispuestos radialmente fue previamente 

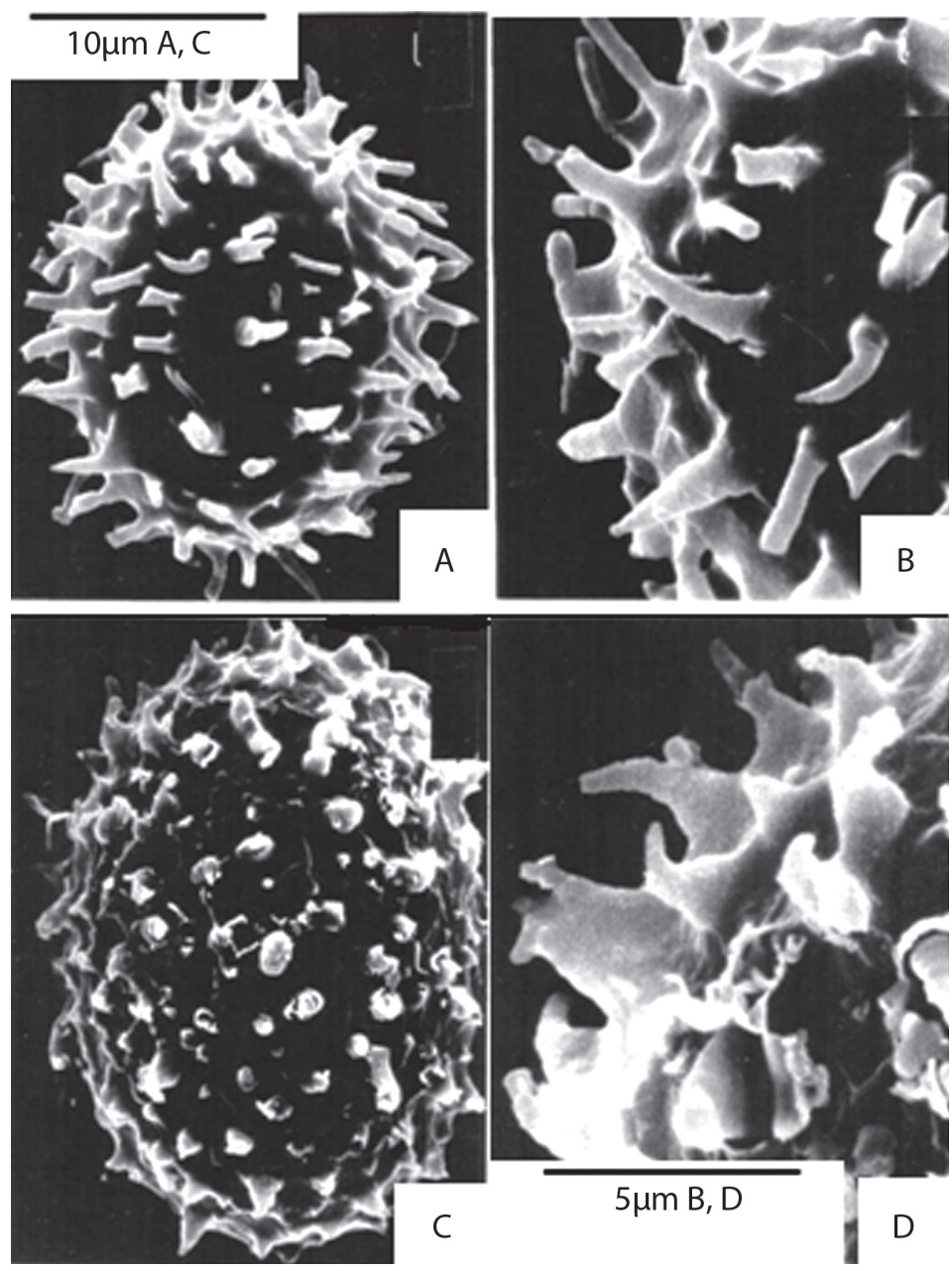

Fig. 4. Esporas de Marattia. (A-B): M. laevis de Costa Rica, Lellinger 1709 (US). (A) Vista polar con perisporio continuo; (B): detalle de espinas (C-D): M. cicutifolia de Brasil, Rohr 1036 (US). (C) Vista polar con perisporio discontinuo. (D) Detalle de espinas. Fig. A, C: x 5000; B, D: x 10000.

Fig. 4. Spores of Marattia. (A-B): M. laevis of Costa Rica, Lellinger 1709 (US). (A) Polar view with continuous perispore; (B): detail of the spines. (C-D): M. cicutifolia of Brasil, Rohr 1036 (US). (C) Polar view with discontinuous perispore. (D) Detail of the spines. Fig. A, C: x 5000; B, D: x 10000.

descrita para ejemplares de M. laevis recolectados en el NE de Argentina y S de Brasil (Ponce \& Smith 1998). Este rasgo podría representar una estrategia adaptativa que mejore la dispersión de las esporas o el paso final en la apertura del sinangio.
Con respecto a las esporas, según Tryon \& Lugardon (1991) las esporas monoletes de Marattia, en particular las de M. laevis se encuentran entre las de menor tamaño de los helechos homosporados. Si consideramos los otros géneros de Marattiales que presentan esporas monoletes, lo planteado por estos 

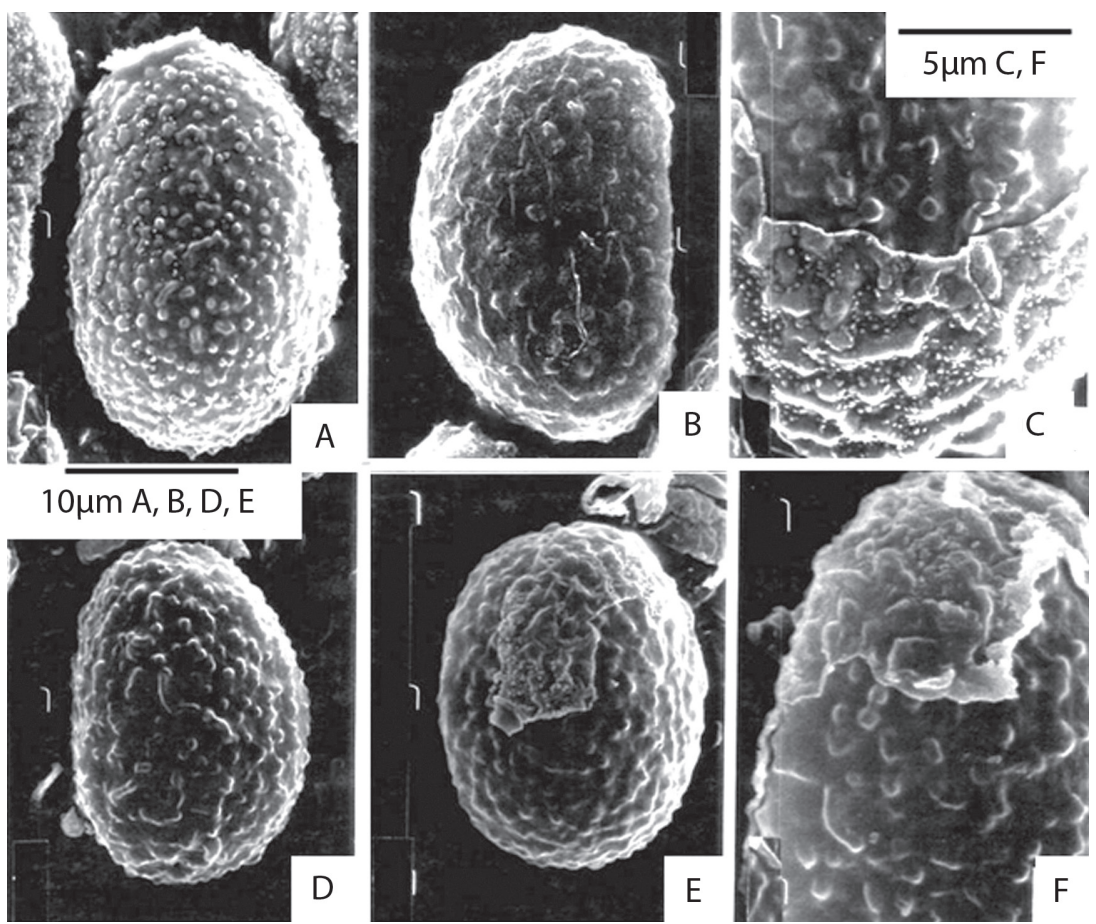

Fig. 5. Esporas de Marattia. (A-C) M. interposita de Panamá, Maxon 5525 (US). (A) Vista polar con exosporio pustulado con micro-ornamentación. (B) Vista proximal con perisporio pustulado-rugado con micro-ornamentación. (C) Detalle de exosporio y perisporio. (D-F) M. alata de Jamaica, Hopkins s/n (1381800 F) y Proctor 3735 (IJ). (D) Vista polar con exosporio pustulado. (E-F) Exosporio y perisporio delgado con micro-ornamentación granular. Fig. A, B, D, E: x 5000; C, F: $x 10000$.

Fig. 5. Spores of Marattia. (A-C) M. interposita of Panamá, Maxon 5525 (US). (A) Polar view with pustulate exospore with micro-ornamentation. (B) Proximal view with pustulate-rugate perispore with micro-ornamentation. (C) Exospore and perispore. (D-F) M. alata of Jamaica, Hopkins s/n (1381800 F) and Proctor 3735 (IJ). (D) Polar view with pustulate exospores. (E-F) Exospore and thin perispore with granular micro-ornamentation. Fig. A, B, D, E: x 5000; C, F: x 10000.

autores concuerda con los resultados de Rolleri et al (1996) respecto de todas las especies de Christensenia; pero no con los de algunas de las especies de Danaea, ya que en D. carillensis H. Christ y D. imbricata Tuomisto \& R. Moran, las dimensiones de las mismas son menores, $17 \mu \mathrm{m}$ x $17 \mu \mathrm{m}$ y $17 \mu \mathrm{m}$ x $16 \mu \mathrm{m}$, respectivamente (Rolleri 2004). Analizando las variaciones intragenéricas, las esporas de $M$. laevis, si bien son pequeñas, no presentan las menores dimensiones entre las especies neotropicales (Cuadro 1) ni entre las paleotropicales (Rolleri \& Lavalle 2001).

La existencia de dos tipos de perisporio en esporas adultas normales producidas por el mismo sinangio, como sucede en $M$. weinmanniifolia, también ha sido registrado para $M$. fraxinea (Rolleri \& Lavalle 2001).

La densidad y altura de las bulas de las esporas pustuladas resultan caracteres diagnósticos adicionales en la diferenciación de esporas en especies paleotropicales del género (Rolleri \& Lavalle 2001); sin embargo en las especies aquí estudiadas esto no es posible debido a que las bulas son extremadamente uniformes y no varía de modo apreciable su densidad.

A continuación se lista una serie de conclusiones respecto de las esporas y estructuras esporógenas de las especies neotropicales de Marattia . 

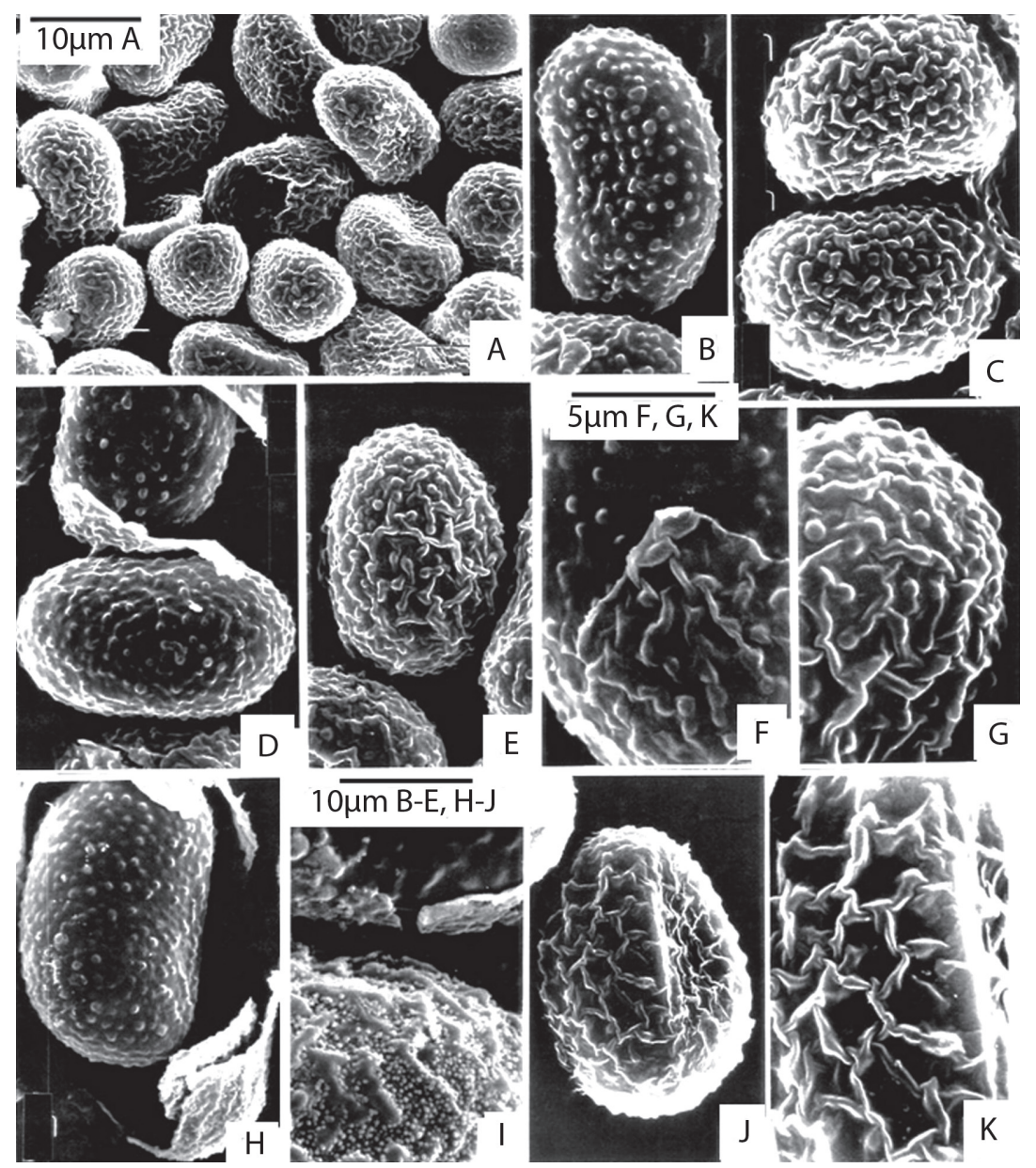

Fig. 6. Esporas de Marattia. (A-C) M. excavata de Guatemala, Proctor 25261 (IJ). (A) Panorámica de varias esporas. (B): Vista polar con exosporio pustulado. (C) Vista polar con perisporio rugado. (D-G) M. laxa de México, Castillo \& Vásquez 1528 (F). (D) Vista polar con exosporio pustulado (E) Vista polar con perisporio pustulado rugado. (F) Detalle de exosporio y perisporio. (G) Detalle de perisporio. (H-K) M. weinmanniifolia de México, Roe et al. 1238 (MICH). (H) vista lateral, exosporio pustulado y restos de perisporio, (I) Detalle de perisporio rugado con micro-ornamentación granular. (J) vista proximal con perisporio retiado. (K) detalle de perisporio y lesura. Fig. A: x 4000; B-E, H-J: x 5000; F-G, K: x 10000.

Fig. 6. Spores of Marattia. (A-C) M. excavata of Guatemala, Proctor 25261 (IJ). (A) Panoramic view of some spores. (B) Polar view with pustulate exospores. (C) Polar view with rugate perispore. (D-G) M. laxa of Mexico, Castillo \& Vásquez 1528 (F). (D) Polar view with pustulate exospores. (E) Polar view with pustulate rugate perispore. (F) Detail of exospore and perispore. (G) Detail of perispore. (H-K) M. weinmanniifolia of Mexico, Roe et al. 1238 (MICH). (H) Lateral view, pustulate exospore and rest of perispore. (I) Detail of rugate perispore with granulate micro-ornamentation. (J) Proximal face with retate perispore. (K) Detail of perispore and laesura. Fig. A: x 4000; B-E, H-J: x 5000; F-G, K: x 10000.

La presencia de sinangios elipsoidales superficiales y de esporas monoletes son rasgos genéricos. En el nivel específico, son caracteres diagnósticos los tipos de micro-ornamentación y macro-ornamentación en exosporios y perisporios.
Los receptáculos suculentos hiperdesarrollados ("pedicelos"), cortos o largos, son característicos de $M$. cicutifolia y M. laevis, y los receptáculos con forma de domos bajos, de las especies neotropicales restantes y de las paleotropicales. 
Las estructuras esporógenas son sinangios superficiales de forma elipsoidal con 10-20 eusporangios fusionados. Por su posición en las láminas, son intramarginales en $M$. alata, $M$. excavata, $M$. interposita y $M$. laxa; y supramediales en $M$. cicutifolia, M. laevis y M. weinmanniifolia.

La ornamentación del exoporio de las especies estudiadas es buliforme (esporas pustuladas), esteliforme (esporas equinadas), y menos frecuentemente muriforme (esporas rugadas y retiadas); en cambio, en las especies paleotropicales no aparecen esporas retiadas ni equinadas.

Los tipos pustulados son similares a los observados en Angiopteris. Los tipos equinados, a los de Archangiopteris, Christensenia y Danaea, aunque la variación morfológica de las espinas es un rasgo genérico. Así las esporas de Marattia tienen espinas cónicas delgadas más bien lisas; las de Christensenia son cónicas irregulares marcadamente anilladas y las de Danaea son basalmente fusionadas, lisas y con micro-ornamentación granular en los ápices.

\section{RESUMEN}

La familia Marattiaceae, que incluye de cuatro a seis géneros, presenta estructuras esporógenas de dos tipos: sinangios en Christensenia, Danaea y Marattia; y soros con eusporangios libres en Angiopteris y Archangiopteris. Marattia es un género pantropical con unas ocho-diez especies en el paleotrópico y siete-ocho en el neotrópico. Mediante MEB se estudiaron las esporas de las siete especies neotropicales de Marattia: M. alata, $M$. cicutifolia, M.excavata, $M$. interposita, M. laevis, $M$. laxa y $M$. weinmanniifolia, basado en la forma de los receptáculos y la posición de los sinangios en material proveniente de distintas colecciones. Los receptáculos son hiperdesarrollados en $M$. cicutifolia y M. laevis, y apenas sobreelevados en el resto de las especies. Los sinangios son elipsoidales y la posición en la lámina es intramarginal a medial. Las esporas son elípticas, siempre monoletes y no se encuentran esporas triletes, abortadas o deformadas. La lesura tiene una longitud aproximadamente igual a la mitad del diámetro de la espora. El perisporio aparece como una capa delgada continua que se deposita siguiendo los procesos del exosporio, en $M$. cicutifolia y $M$. laevis. Es liso en $M$. alata, rugado en $M$. excavata y pustulado-rugado en dos especies: $M$. interposita y $M$. laxa. El exosporio es equinado en $M$. cicutifolia y $M$. laevis, y pustulado en las otras especies. En M. weinmanniifolia, las esporas producidas por el mismo sinangio tienen diferentes tipos de ornamentación. La presencia de sinangios elipsoidales superficiales y de esporas monoletes, son rasgos genéricos; mientras que los tipos de micro-ornamentación y macroornamentación en exosporios y perisporios son caracteres diagnósticos específicos. La macro-ornamentación puede ser buliforme (pustulada), una combinación de buliforme y muriforme (pustulada-rugada), muriforme (rugada-retiada) y esteliforme (equinada); es frecuente la presencia de micro-ornamentación granular en el perisporio.

Palabras clave: esporas, Marattia, neotrópico, ornamentación, receptáculos, sinangio.

\section{REFERENCIAS}

Bierhorst, D.W. 1971. Morphology of vascular plants. Macmillan, Nueva York, EEUU.

Christenhusz, M.J.M. 2007. Evolutionary history and taxonomy of Neotropical marattioid ferns: Studies of an ancient lineage of plants. Annales Universitatis Turkuensis, Sarja - Ser. AII Osa - Tom. 216, Turku, Finlandia.

Copeland, E.B. 1947. Genera Filicum. The genera of ferns. Waltham Chronica Botanica, Annales Cryptogamici et Phytopathologici $\mathrm{N}^{\circ} 5$.

D’ambrogio de Argüeso, A. 1986. Manual de técnicas en histología vegetal. Hemisferio Sur. S.A., Buenos Aires, Argentina.

Gurr, E. 1965. The rational use of dyes in biology. Leonard Hill, Londres, Gran Bretaña.

Hill, C.R. \& J.M. Camus. 1986. Evolutionary cladistics of marattialean ferns. Bull. Brit. Mus. (Nat. Hist.), Bot. 14: 219-300.

Holmgren, P.K., N.H. Holmgren \& L. Barnett. 1990. Index Herbariorum, Part I, Herbaria of the World. New York Botanical Garden, Bronx, Nueva York, EEUU.

Lavalle, M.C. 2003. Taxonomía de las especies neotropicales de Marattia Sw. (Marattiaceae). Darwiniana 41: 61-86.

Lavalle, M. C. 2005. Arquitectura foliar y otros caracteres del esporófito en especies neotropicales de Marattia Sw. (Marattiaceae-Pteridophytas). Acta Bot. Malacitana 30: 11-30.

Lavalle, M.C. 2007. Caracteres diagnósticos foliares en especies neotropicales de Marattia Sw. (Marattiaceae-Pteridophytas). Ann. Missouri Bot. Gard. 94: 192-201. 
Lavalle, M.C. \& A. Mengascini. 2007. Almidón de reserva en especies neotropicales de Marattia Sw. (Marattiaceae-Pteridophyta). Anales del Jardín Botánico de Madrid 64: 79-85.

Lavalle, M.C., A. Mengascini \& M. Rodríguez. 2008. Protuberancias intercelulares pécticas en especies neotropicales de Marattia (Marattiaceae-Pteridophyta). Bol. Soc. Argent. Bot. 43: 247-253.

Lellinger, D.B. 2002. A modern multilingual glossary of taxonomic pteridology. Pteridologia 2A: 1-364.

Liu, Zh, Cs Li \& J. Hilton. 2001. Fertile pinnules of Danaeites rigida $\mathrm{Gu} \& \mathrm{Zhi}$ (Marattiales) from the Upper Permian of south China. Bot. J. Linn. Soc. 136: 107-117.

Millay, M.A. 1978. Studies of Paleozoic marattialeans: The morphology and phylogenetic position of Eoangiopteris goodii sp. n. Amer. J. Bot. 65: 577-583.

Millay, M.A. 1979. Studies of Paleozoic marattialeans: A monograph of the American species of Scolecopteris. Palaeontographica B 169: 1-69.

Pérez García, B. 1995. Marattiaceae. In R.C. Moran \& R. Riba (eds.). Flora Mesoamericana 1: 50-51. Universidad Nacional Autónoma de México, México.

Ponce, M.M. \& A.R. Smith 1998. Sobre la presencia del género Marattia (Marattiaceae, Pteridophyta) en la Argentina. Darwiniana 35: 179-182.

Rolleri, C. 2004. Revisión del género Danaea (Marattiaceae-Pteridophyta). Darwiniana 42: 217-301.
Rolleri, C. \& M. Lavalle. 2001. Morfología de las esporas de especies paleotropicales de Marattia Sw. (Marattiaceae). Revista Mus. La Plata, n.s., Bot. 14: 695-705.

Rolleri, C., M. Lavalle, A. Mengascini \& M. Rodríguez. 1996. Spore morphology and systematics in the genus Christensenia. Amer. Fern J. 86: 80-88.

Rolleri, C., M. Lavalle, A. Mengascini \& M. Rodríguez. 1998. Morfología de las esporas en Angiopteris Hoffm. y otras Marattiales. Physis (Buenos Aires), Sec. C 55: 17-26.

Rolleri, C., M. Lavalle, A. Mengascini \& M. Rodríguez. 2001. El género Marattia Sw. (Marattiales, Marattiaceae) en el paleotrópico. Candollea 56: 97-113.

Rolleri, C., M. Lavalle, A. Mengascini \& M. Rodríguez. 2003. Sistemática de los helechos maratiáceos. Rev. Mus. La Plata, Bot. 15: 23-49.

Smith, J.E. 1842. An arrangement and definition of the genera of ferns with observations on the affinities of each genus. Lond. J. Bot. 1: 419-659.

Smith, A., K. Pryer, E. Schuettpelz, P. Korall, H. Schneider \& P. Wolf. 2006. A classification for extant ferns. Taxon 55: 705-731.

Taylor, T.N. 1981. Paleobotany: An introduction to fossil plant biology. McGraw-Hill, Nueva York, EEUU.

Tryon, A.F. \& B. Lugardon. 1991. Spores of the Pteridophyta. Springer Verlag, Nueva York, EEUU. 